\title{
GENERIC BI-LAYERED NET PROGRAMMING
} General Software for the Simulation of Hybrid Processes

\author{
Béla Csukás, Sándor Balogh, Gyöngyi Bánkuti \\ Institute of Mathematics and Information Technology, University of Kaposvár, Guba S. u. 40., \\ Kaposvár, 7400, Hungary; Process Information Techn. Ltd., Bárd u. 26., Bárdudvarnok, \\ 7478,Hungary; csukas@matinf.gtk.u-kaposvar.hu; bankuti@matinf.mail.gtk.u-kaposvar.hu; \\ balogh(matinf.gtk.u-kaposvar.hu
}

Abstract: $\quad$ Based on the Generic Bi-layered Net model, an effective software architecture has been developed for the Direct Computer Mapping of process models. The novel, model-driven programming technique makes possible the natural, model-driven software generation for the simulation based problem solving. The PROLOG implementation of the GBN model has been applied for the solution of various difficult practical problems. The solution supports the parallelism of different granularity.

Key words: programming technique, software architecture, Direct Computer Mapping, Generic Bi-layered Net, hybrid processes, dynamic simulation, Prolog

\section{INTRODUCTION}

Many recently used engineering methods had been established before the onset of powerful computers. Modeling starts either from the consideration of changes in characteristic measures, or from the rules and signs. Next this is transformed into mathematical construct. It usually cannot be solved, should be discretisized and, finally, the computer executes simple arithmetical steps. However, mostly there is not a plausible connection between the final computer operations and the primary functioning of the building blocks, directly.

In Direct Computer Mapping [1] we can map the simple building blocks of the conservational and informational processes onto the generic "active" and "passive" elements of an executable program. The balance 
elements and the signs, as well as the elementary transitions and the rules can be described by brief uniform programs, executed by the same kernel algorithm. Direct Computer Mapping of process models allows the computer to know explicitly about the very structures and bounds of the physical world. In this knowledge representation, the model is organized rather by the transitions, than by the state. The key issue is that the computational software (and/or optional hypothetical hardware) can copy the natural structure and building elements of the investigated problem.

\section{THE GENERIC BI-LAYERED NET SOFTWARE ARCHITECTURE}

In the software implementation of the $\langle P, A, B, G, X, Y, \Phi, \Psi, \underline{r}, t\rangle$ tentuplet of the Generic Bi-layered Net model [2], the dynamic partitions of the elements and connections are executed by a general and optionally extendable kernel.

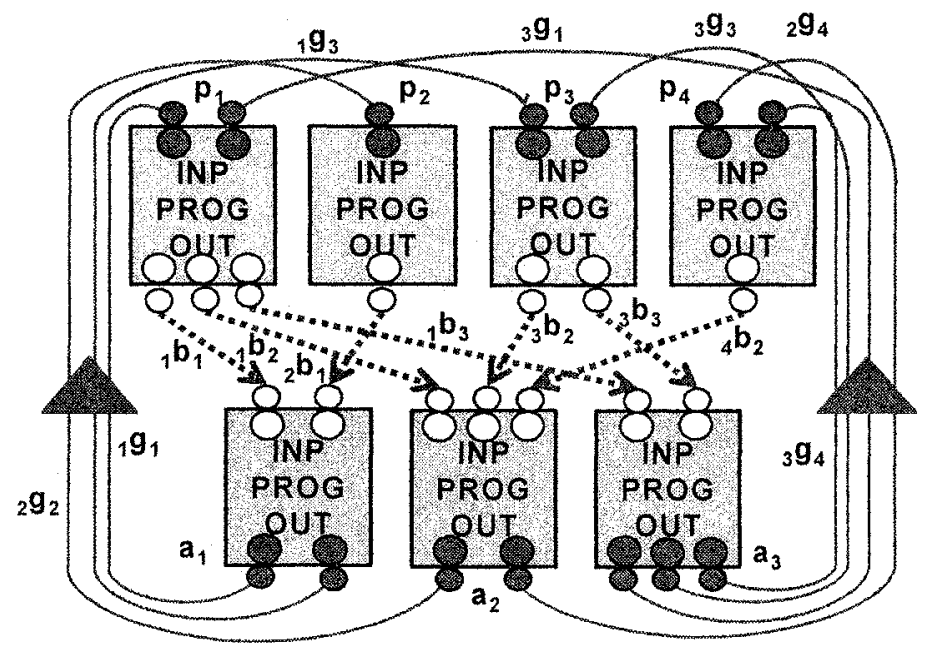

Figure 1. The GBN model based software architecture

According to Fig.1, the execution consists of four, cyclically repeated consecutive steps, as follows:

(1) active elements $\mathrm{A}$ read the content $\mathrm{X}$ from the associated passive elements according to the reading channels $\mathrm{B}$;

(2) programs $\Phi$, associated with elements A calculate the changes;

(3) passive elements $P$ are changed according to $Y$ changes carried via modifying channels $\mathrm{G}$;

(4) operators $\Psi$, associated with the elements P calculate the new state. 
The first complete implementation of GBN architecture is written in PROLOG, where the free unification of the high level structures contained in dynamic partitions supports the methodology.

In the PROLOG representation, the elements of sets $P, A, B$ and $G$ correspond to the automatically generated dynamic partitions, describing the actual detailed state, the changes, the readings and the modifications of the modeled process. Accordingly variables $X$ and $Y$ correspond to the freely determined possible set of signs transported via the communication channels $\mathrm{B}$ and $\mathrm{G}$. The fundamental predicates are the following:

p ( PID, Name, Space, Time, Glist, PassiveID, State, Blist )

b ( BID, PID, X, AID )

a ( AID, Name, ISpace, OSpace, Time, Blist, ActiveID, Process, Glist) g ( GID, AID, Y, PID )

where:

PID, BID, AID, GID = the identifiers of the passive elements, reading channels, active elements and modifying channels, respectively;

Name $\{$ stringlist $\}=$ the name of the element (in hierarchical models the name describes the subsequent elements of the tree);

Space $\{$ integerlist $\}=$ the spatial and property coordinates in the case of the processes with distributed parameter);

Time $\{$ tlist $\}=$ the temporal existence of the elements according to variable $\tau$, declared by the Time $=[\operatorname{tk}(\mathrm{S}, \mathrm{E},[\mathrm{T} 1, \ldots, \mathrm{TN}], \mathrm{DT}, 0), \ldots]$ data structure, where $\mathrm{S}$ and $\mathrm{E}$ are the starting and ending points of the time intervals, as well as $\mathrm{T} 1, \ldots, \mathrm{TN}$ are the discrete points of time, when the given element is to be taken into consideration, while DT is the optional time step of the repeated execution;

Blist $\{$ blist $\}=$ the list of (feedback) readings, determining the input data for the functioning of the active elements, i.e. blist $=b^{*}$;

Glist $\{$ glist $\}=$ the list of changing, corresponding to the output data of the active elements, i.e. glist $=\mathrm{g}^{*}$;

$\mathrm{X}\{$ sign $\}=\mathrm{a}$ user configured data set about the state (e.g. integers, reals, strings, tables, fuzzy variables, optional records, etc.);

$\mathrm{Y}\{$ sign $\}=\mathrm{a}$ user configured data set describing an individual change (increase, decrease, overwriting, modification, etc.);

State $\{$ state_data $\}=$ a user configured static and/or dynamic data set characterizing the state of a given class of passive elements;

Process \{process_data $\}=$ a user configured static and/or dynamic data set, associated with a given class of active elements;

PassiveID $=$ identifier of the dynamic clause partition $\psi$ that executes the functionality associated with the given passive element;

ActiveID $=$ identifier of the dynamic clause partition $\varphi$ that executes the functionality associated with the given active element. 
The $\Psi$ and $\Phi$ functionalities are described by the dynamic partitions of clauses

passive( PassiveID, Glist, State, Blist ) if

$\ldots$ and

active( ActiveID, Blist, Process, Glist ) if

..., respectively.

\section{APPLICATIONS OF THE METHODOLOGY}

All of the listed adaptations run with the same kernel program, supplied with the respective domain specific interpreter module. Actually only a single one of the 12 modules has to be modified when moving from one application to the other. As an actual challenge, the present efforts are concentrated on developing a platform independent code for any, arbitrary application, where the domain specific structures and functionalities can be edited by the field expert, using the so-called expert's interface.

\subsection{Model Based Design and Control of Cyclic, Pseudo- steady State (SMB) Separation Processes}

Practical problem to be solved: Design and control of the countercurrent separation processes, realized in the so-called Simulated Moving Bed (SMB) system. The key idea of SMB is to "simulate" the solid phase motion by periodic switching the inlet and outlet ports of the unit.

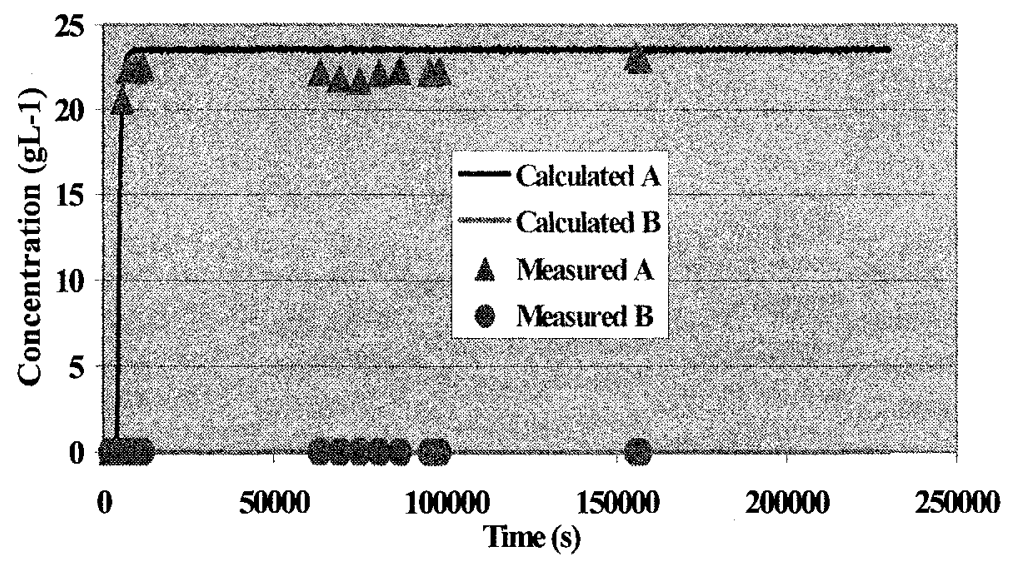

Figure 2. Measured and calculated data of an SMB fraction 


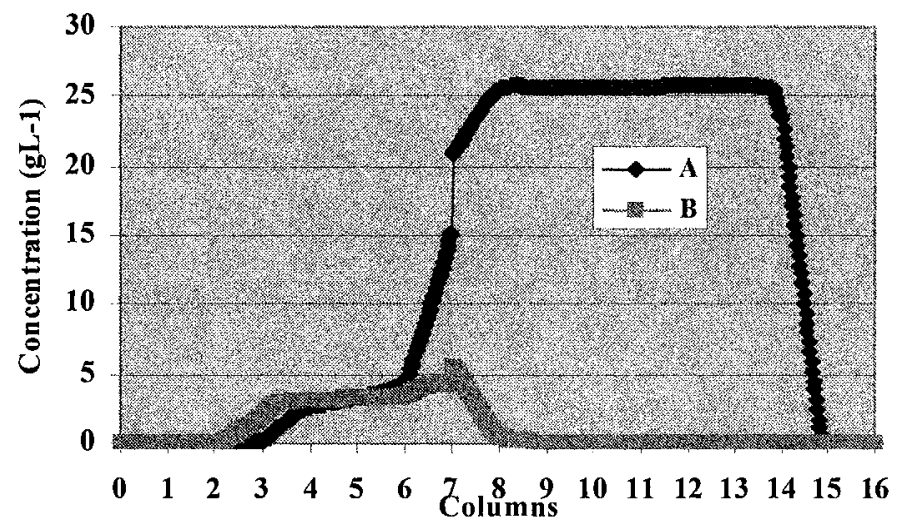

Figure 3. Calculated concentration profile of the SMB

Passive elements (P) Individual competitive components in the given phase at a given compartment along the length of the respective column.

Active elements $(A)$ : The terminal elements designate the architecture of the elementary transformations, transportations, and rules (control actions).

State functionalities ( $\Psi$ ): These brief dynamic program partitions define the calculation of the intensive properties from the extensive ones.

Transition functionalities $(\Phi)$ : These brief dynamic program partitions declare the calculation of the component transfer, of the liquid flow, etc. for the conservational processes, as well as the execution of the diagnostic, control and evaluating rules.

Input data: The user has to define the configuration of the system, the data of the columns, the initial state, the equilibrium and the process parameters (flow rates, column switch time, etc.). The kinetic and mixing parameters have to be identified from elution tests, using the simulator itself.

Output results: There are detailed time functions for the instantaneous and column step averaged concentrations (see Fig. 2.), as well as for the concentration profiles (see Fig. 3.) along the length of the column series.

Experiences: There is a good agreement between the measured and calculated results (see Fig. 2.). The simulator helped in the successful design and control of SMB processes in the pharmaceutical industry [3].

\subsection{Identification of Cellular Metabolic Processes}

Practical problem to be solved: There is a gap between the genomic information discovered by bioinformatics and the organization \& functioning of the metabolic processes. The efforts to synthesize the major 
cellular functions from the in vitro measured individual enzyme kinetics failed. Biosystem engineers need large, complex models, built from the elementary steps. The models must be able to be supplemented with any new interaction (e.g. in drug discovery or in toxicology). These models have to connect the metabolism with the polypeptide formation and degradation, too.

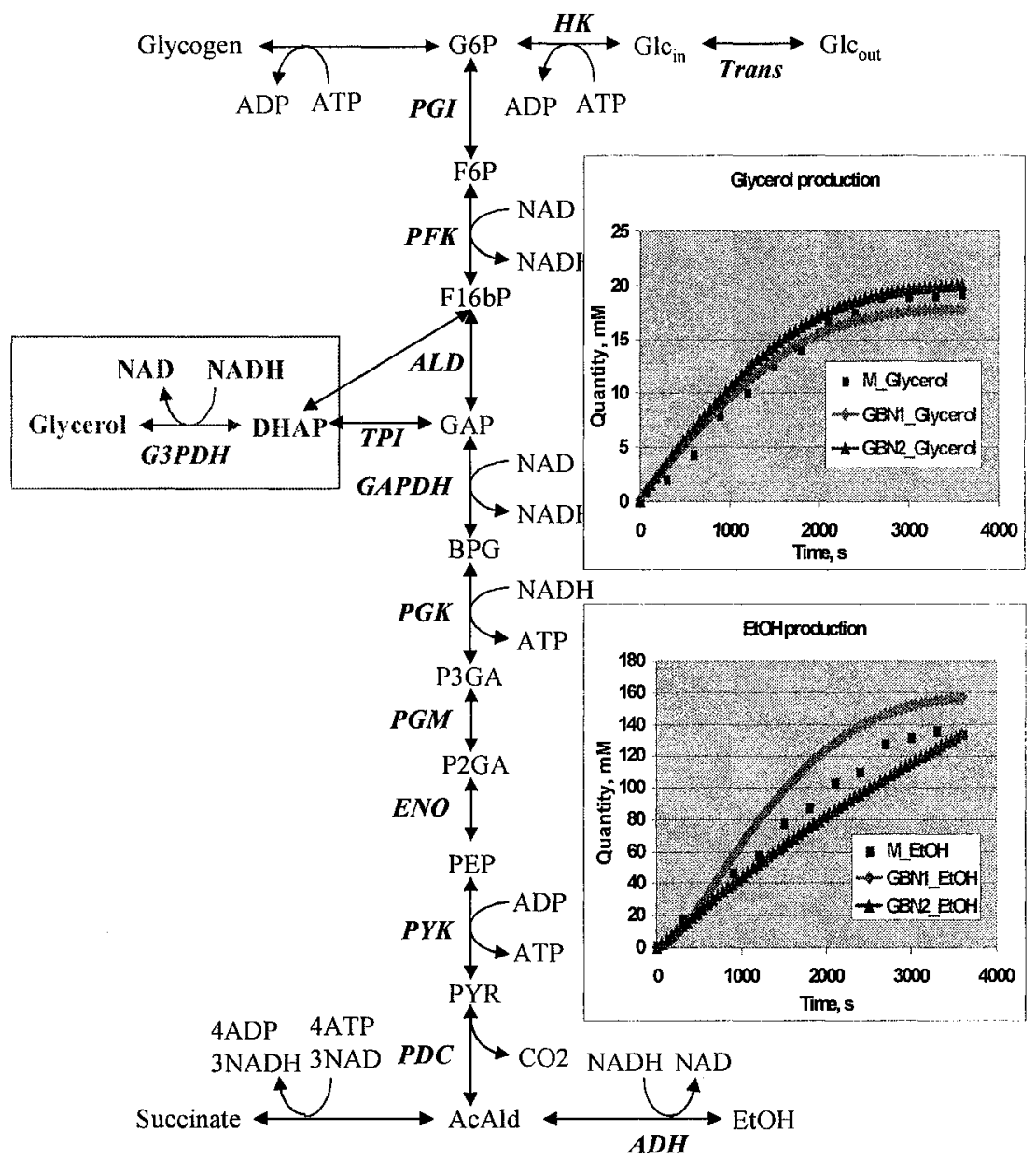

Figure 4. Measured and calculated data of glycolysis

Special difficulties of the simulation: Regardless to the equilibrium reaction kinetic approach of biochemistry, the chemical engineer's way of thinking has to be combined with the discrete cellular machineries, working as the production lines in mechanical engineering. The biosystems are highly underdetermined and, the useable models need special identifying abilities to treat the missing or uncertain knowledge. 
Passive elements (P) All of the possible, more or less freely moving compounds and complexes, as well as the structured, located sites that may have any functionality in the various compartments.

Active elements (A): All of the possible elementary transformations inside the compartments, and transportations between the compartments. State functionalities $(\Psi)$ : These brief dynamic program partitions define the calculation of the intensive properties from the extensive quantities, as well as the evaluation of the signs for the control systems.

Transition functionalities $(\Phi)$ : These brief dynamic program partitions declare the calculation of the transformations and transportations of the conservational processes, as well as the execution of the control functions in the signaling processes.

Input data: The available quantities and concentrations, as well as the estimations of the process parameters. The detailed equilibrium, kinetic, transporting and signaling parameters have to be identified from possible measurements, using the simulator combined with a genetic algorithm [4].

Output results: The calculated change in the amount and concentration of the various metabolites and macromolecular complexes, as well as the state of the keynote cellular elements.

Experiences: There is a good agreement between the measured and calculated results (see Fig. 4.) of the yeast glycolysis [5].

\subsection{Quantitative Health Risk Analysis of Multiproduct Batch Plants}

Practical problem to be solved: Health risk management at multiproduct workplaces is a typical field, where computer simulation can help to cope with strict and sometimes ambiguous regulations. Standards do not contain proper methodologies for handling the presence of multiple inhalative risk sources, distributed in space and time, according to the dynamic changes in production demand and schedule.

Special difficulties of the simulation: The various sources are allocated in interconnected workplaces with multiple ventilation inputs and outputs, while employees are working in the vicinity of different sources of potential inhalative risk.

Passive elements (P) Various compounds in the compartments of the plant building, equipments, ventilation units, workers, etc.

Active elements (A): Loss of solvents, sources of the dangerous compounds, input / output arrangement of the risk factor calculation, etc.

State functionalities $(\Psi)$ : Calculation of balances, intensive parameters, equilibrium properties, etc. 
Transition functionalities $(\Phi)$ : Calculation of the losses, flows, risk factors, etc.

Input data: First the equipments, the partially open ceilings and walls, as well as the ventilation system must be allocated in the simplified, discretisized 3D representation of the plant building, where the various batch technologies are running simultaneously. Next, with the knowledge of the actual (daily, weekly) schedule, the elementary models of the events, associated with potential sources of inhalative exposure risk are generated from the SQL database, comprising all of the data related to equipments, process steps and materials. Finally, considering the shift schedule, the employees are allocated to the safe and potentially risky places, while the use of personal protective equipment may be also taken into account.

Output results: The method makes possible the detailed calculation of the risk factors of the employees. The tool is also able to simulate the effect of any feature (e.g. modified ventilation characteristics, changed substances or duration of different procedures) on the inhalative risk. A simplified illustration [6] can be seen in Fig. 5.

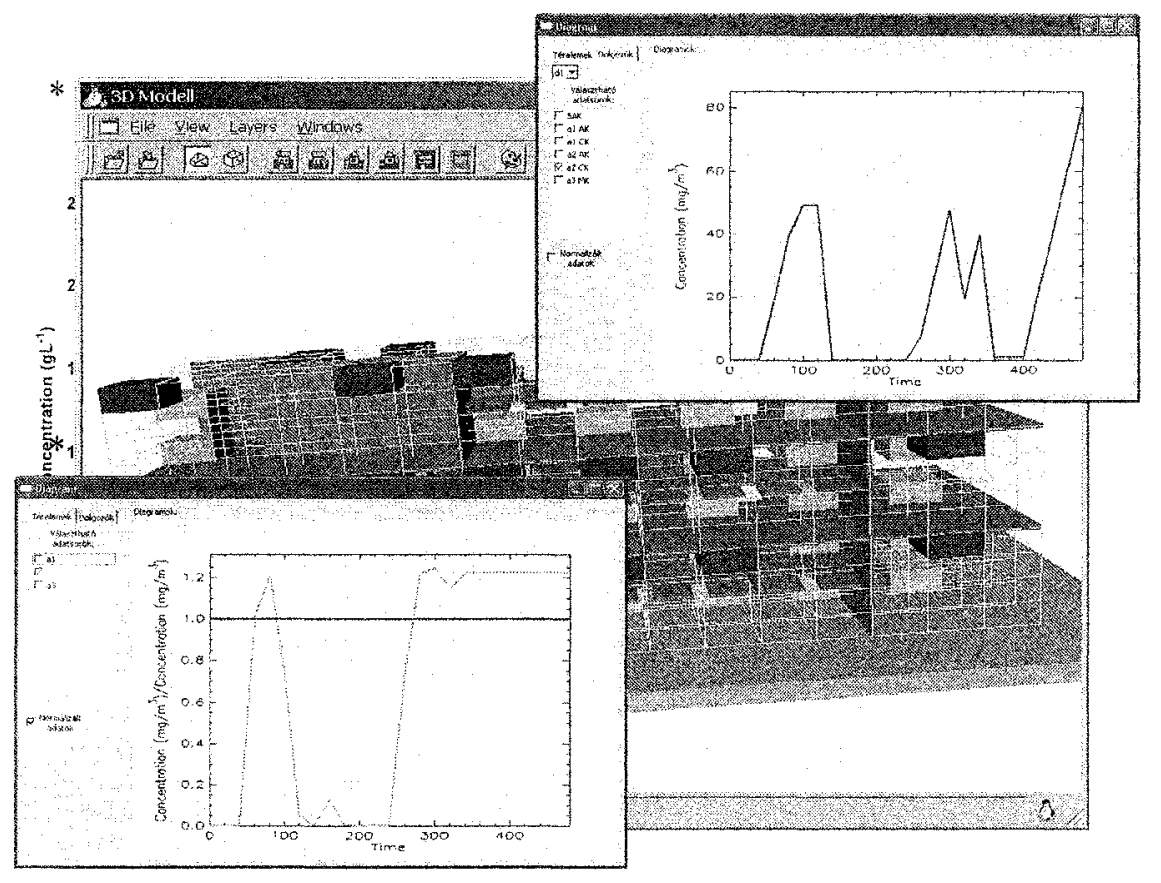

Figure 5. Illustration of the simulation model

Experiences: Detailed characterization of the losses and risk can be solved with considerably less number of expensive analytical measurements. 


\subsection{Simulation Based Cash Flow Analysis of an Agricultural Farm}

Practical problem to be solved: Planning and scheduling of an agricultural farm, including the liquidity (cash flow) analysis.

Special difficulties of the simulation: There is a lot of elaborated data in form of statistical correlations, but the consistent system of the first principle expressions for the appropriate balance equations has not evolved yet. In addition, the qualitative knowledge of the field expert has not collected in a systematic way.

Passive elements (P) There are various groups of realistic and fictitious storage volumes at vendors, inside the farm, as well as at the consumers, containing seeds, crops, fertilizers, etc. Another class of passive elements declares the manpower, the machine resources and the economic properties.

Active elements (A): Describe the structure of the possible purchases, sales, as well as of the agricultural processes (e.g. ploughing, harrowing, sowing, harvest, etc.).

State functionalities $(\Psi)$ : Describe the calculation, associated with the storages and evaluation of the signs for the control system.

Transition functionalities $(\Phi)$ : Determine the simulation of the agricultural processes (consumption of land, seeds, crops, fertilizers, chemicals, machines, manpower and the productions, as well as calculate the economic characteristics.

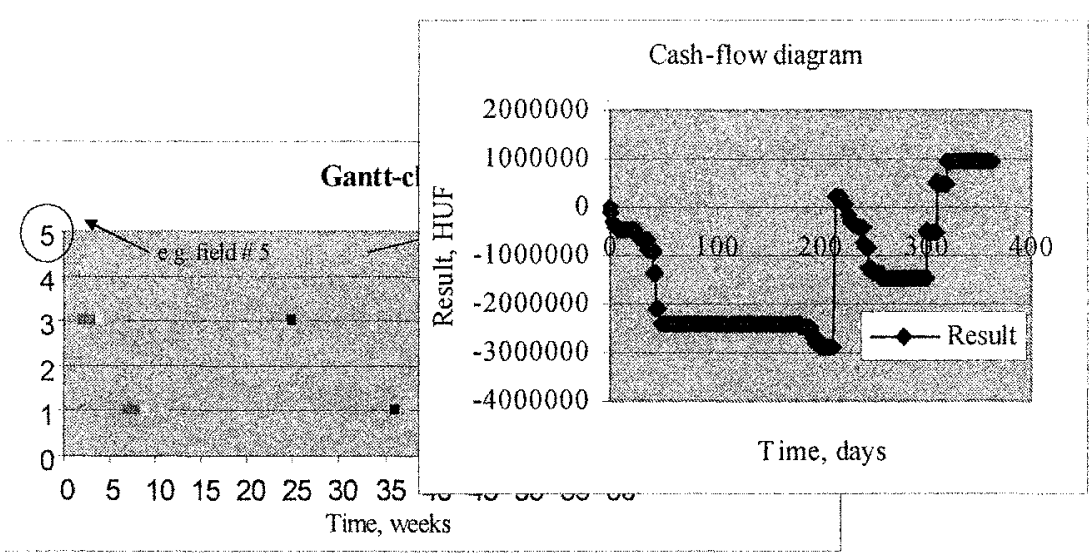

Figure 7. Simulation of an agricultural farm

Input data: The user has to define the initial state of the agricultural system, the estimated (optionally changing) economical parameters, as well 
as the possible, alternative agricultural activities, described by the sequence of the respective operations.

Output results: The simulated output describes the detailed change of the characteristic materials, energy, manpower and machine consumption, as well as the cash-flow of the alternative plans. In addition, the Gantt-chart of the allocated resources can also be generated (see Fig. 7).

Experiences: The calculated results are in good agreement with the practical experiences and, having connected the simulator with a genetic algorithm, feasible propositions for more favorable rotations of crops have been suggested [7].

\section{ACKNOWLEDGEMENTS}

The work was supported by Hungarian Scientific Research Fund T 037-297, by the Gedeon Richter Ltd., and by the Grant of Hungarian Ministry of Economy and Transport GVOP-3.3.1 200404-0091/3.0.

\section{REFERENCES}

1. Csukas B.: Direct Computer Mapping of Conservational and Informational Processes, DSc Thesis, Manuscript, 2001 (in Hungarian).

2. Bánkuti Gy., Csukás B.: Generic Bi-layered Net Model - General Methodology for Process Simulation, Proceedings of the Second IFIP Conference on Artificial Intelligence Applications and innovations, Beijing, China, September 7-9, 2005.

3. Temesvári K., Aranyi A., Csukás B., Balogh S.: Simulated Moving Bed Separation of a Two Components Steroid Mixture. (Accepted manuscript for Chromatographia, 2003).

4. Csukás B., Balogh S.: Combining Genetic Programming with Generic Simulation Models in Evolutionary Synthesis. Computers in Industry, 36, 181-197 (1998).

5. Csukás B., Debelak, K. A., Prokop, A, Balcarcel, R. R., Tanner, R. D., Bánkuti Gy, Balogh S.: Generic Bi-layered Net Model Based Discrimination of Chemical and Biological Warfare Agents, AIChE Annual Meeting, San Francisco, November 16-20, 2003, Manuscript $474 f$.

6. Balogh S., Négyesi Gy., Budai M., Sógor A., Csukás B.: Measurement and Dynamic Simulation of Solvent Exposure in Multiproduct Batch Plants. Process Engineering Days' 2003, Veszprém, April 8-10, pp. 284-290, 2003 (in Hungarian).

7. Bosnyák B., Balogh S., Csukás B.: Planning and Scheduling of a Plant Cultivating Farm by means of Generic Simulation and Genetic Algorithm, 4-th Conference on Applied Informatics, Kaposvár, May 27, 2005 (in Hungarian). 